\title{
Systemic immunological biomarkers of clinical responses in immune checkpoint blockade therapies
}

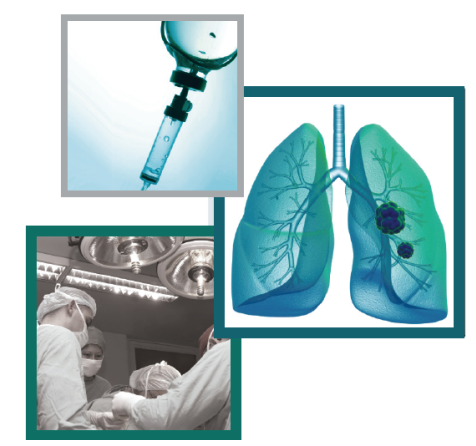

\author{
Hugo Arasanz ${ }^{\ddagger 1,2}$, Miren Zuazo ${ }^{\ddagger} 1$, Ruth Vera*,2, Grazyna Kochan**,1 \& David Escors***,1,3 \\ ${ }^{1}$ Immunomodulation Group, Navarrabiomed-Fundación Miguel Servet, IdISNA, Complejo Hospitalario de Navarra (CHN), Irunlarrea \\ 3, 31008 Pamplona, Navarra, Spain \\ ${ }^{2}$ Department of Oncology, Complejo Hospitalario de Navarra (CHN), Irunlarrea 3, 31008 Pamplona, Navarra, Spain \\ ${ }^{3}$ Division of Infection \& Immunity, Rayne Institute, University College London, 5 University Street, London WC 1E 6JF, UK \\ *Author for correspondence: ruth.vera.garcia@navarra.es \\ **Author for correspondence: grazyna.kochan@navarra.es \\ ***Author for correspondence: descorsm@navarra.es \\ $\ddagger$ Authors contributed equally
}

"The discovery of immune checkpoint interactions as a strategy used by cancer cells to inhibit the antitumor capacities of the immune system, and the development of drugs blocking these interactions, have revolutionized the treatment of lung cancer"

First draft submitted: 15 November 2018; Accepted for publication: 21 November 2018; Published online: 20 December 2018

Keywords: B7-H1 • biomarker • immune checkpoint blockade • NSCLC • PD-1 • PD-L1 • T lymphocyte

The discovery of immune checkpoint interactions as a strategy used by cancer cells to inhibit the antitumor capacities of the immune system, and the development of drugs blocking these interactions, have revolutionized the treatment of lung cancer. In NSCLC, immunotherapy targeting PD-1/PD-L1 interactions has become standard for platinumrefractory patients and for first-line treatment of metastatic tumors. Immune checkpoint blockade is applied as single therapy, in combination with chemotherapy or as consolidation therapy after chemoradiotherapy in locally advanced tumors (KEYNOTE-189) [1]. Some recent data from clinical trials (NCT02763579) [2] demonstrate that immune checkpoint blockade might improve survival of patients with small-cell lung cancer.

Immune checkpoint blockade does extend survival compared with chemotherapy regimens $[1,3]$. However, most of the benefit is hoarded by a small proportion of patients, exhibiting long-term disease control which does not usually go above $20 \%$. Importantly, hyperprogressive disease has been described in a proportion of patients for whom there is an acceleration of tumor growth following immunotherapies [4]. This seems to be especially true for lung cancer. For these reasons, the finding of predictive biomarkers of responses to immune checkpoint blockade constitutes a major research issue of critical importance in oncology. Due to this necessity, extensive research is currently being carried out to identify biomarkers of responses. Most studies utilize 'open' nonbiased approaches for the identification of these biomarkers. This has been strongly promoted by the development of high-throughput analytic techniques such as CyTOF, liquid biopsies and next-generation sequencing (NGS). These techniques allow the simultaneous quantification of multiple parameters and cell lineages. Even the use of machine learning is being applied to identify the relevant biomarkers from vast amounts of data that are not apparent to the day-to-day biomedical researcher and oncologist.

One of the first biomarkers to be tested was PD-L1 tumor expression, as this molecule is a direct target of anti-PD-L1/PD-1 blockade therapies. Indeed, there is a correlation between the percentage of PD-L1-expressing cells and clinical benefit in NSCLC. However, the predictive value of PD-L1 expression varies between drugs and tumor histology. This was apparent in CheckMate 017 and CheckMate 057 for the treatment of squamous and nonsquamous NSCLC [5]. Such variety was even shown with the same drug in similar patient cohorts such as in Phase II POPLAR (NCT01903993) [6] and Phase III OAK (NCT02008227) [7] with atezolizumab. Moreover, no association has been demonstrated between PD-L1 expression and efficacy in small cell lung cancer 
(NCT02763579) [2]. The interpretation is even more challenging when PD-1/PD-L1 checkpoint blockade is combined with chemotherapy or antiangiogenic drugs, as many factors might influence the outcomes. Inconsistency between trials could be explained by the fact that PD-L1 is a dynamic marker, characterized in many instances by heterogeneous expression within the tumors and even in immune infiltrates. In addition, the time elapsed between the biopsy and the start of immunotherapies can cover months and may not represent the current status of the tumors in the patient.

Tumor mutational burden (TMB) is associated with better clinical outcomes [8], although so far the data is still scarce. In fact, TBM was a better predictor than PD-L1 tumor expression for selecting patients undergoing iplimumab/nivolumab combination in Phase III CheckMate 227. However, TMB evaluation is very costly compared to PD-L1 immunohistochemistry when performed through whole-exome sequencing (WES). NGS algorithms have been developed for clinical use, and even a NGS-based in vitro diagnostic test under the name FoundationOne $\mathrm{CDx}^{\top \mathrm{TM}}$ has been approved by the US FDA. Nevertheless, the elevated cost of these techniques appears to be a significant limitation for its routine application.

In the last decade, immune checkpoint blockade-based immunotherapy has caused a change of paradigm in oncology. Now the target of the treatments is fundamentally the immune system and not solely the tumor. Hence, the components and regulatory mechanisms of the immune system will deeply condition the efficacy. This has been previously shown in numerous preclinical immunological studies before immune checkpoint blockade was applied in human therapy. It is reasonable to revise all previous extensive work on cancer immunotherapy for cherished predictive biomarkers or clues of what these biomarkers might be. Some efforts have been put toward this goal. The quantification of tumor infiltrating lymphocytes as a prognostic biomarker has been known for decades. Indeed, more technically developed methods based on tumor infiltrating lymphocyte (and other immune cell infiltrates) quantification have been developed for predicting clinical outputs in immune checkpoint blockade therapies. The immunoscore is probably one of the most widely used methods [8,9]. However, the technical difficulties in attaining a precise and reproducible evaluation strongly limits its application. Undoubtedly, the use of systemic biomarkers would ease up their routine clinical use, particularly those quantified from small peripheral blood samples. The neutrophil-to-lymphocyte ratio has also been known for several years to be a prognostic biomarker for many cancer types. A meta-analysis study revealed the utility of neutrophil-to-lymphocyte ratio as a prognostic biomarker in NSCLC, but not so much for small-cell lung cancer [10]. The development of high-throughput analytical techniques allows for the more precise quantification of immune cell populations. Multicolor flow cytometry is inexpensive, and can be translated easily to routine clinical practice. An elegant translational work by Kamphorst et al. showed that CD8 CD28 T-lymphocyte expansion occurred in patients who responded to anti-PD-1 therapy [11]. This is a demonstration of a dynamic direct activity of anti-PD-1 antibodies over systemic T-cell populations. Another high-throughput study from peripheral blood using CyTOF and machine learning showed that responder patients had increased baseline percentages of $\mathrm{CD} 14^{+}$monocytes [12]. However, the differences between nonresponder and responder patients were minimal and possibly cannot be detected by standard approaches. In addition, the very high cost of CyTOF makes it difficult for routine clinical use at the moment.

Our group has been interested in the study of fundamental immunological anticancer mechanisms for several years, particularly the role of PD-L1/PD-1 interactions on cancer cells and T-cell responses $[13,14]$. The role of CD4 T-cell responses in antitumor immunity has been controversial over the decades but most of the preclinical evidence highlights their key contribution. Let us not forget that CD4 T cells are main regulators of CD8 T cells during antigen presentation. Recent data is confirming the importance of neoantigen-specific CD4 $\mathrm{T}$ cells for efficacious antitumor immunity [15]. In a recent work, we have monitored the dynamics of different CD4 T-cell populations systemically in peripheral blood of NSCLC patients who are undergoing anti-PD-L1/PD-1 therapies as second and third-line treatment. Interestingly, we found that patients could be divided in two groups according to the baseline percentage of highly differentiated memory CD4 T cells [16]. Patients with low percentages of this CD4 T-cell subset before the start of therapy did not show objective responses to PD-1/PD-L1 immunotherapy. Indeed, the overall response rate was of $0 \%$. In contrast, patients with high baseline percentages of memory CD4 T cells showed overall response rates of $50 \%$, and close to $70 \%-80 \%$ when combined with PD-L1 positivity. Hence, CD4 T-cells may be playing a more direct role in the efficacy of anti-PD-L1/PD-1 immunotherapies than previously anticipated.

Indeed, dynamic changes of CD4 T-cell populations could be successfully used for 'real time' monitoring of responses from small blood samples during immunotherapies. Information on dynamic changes in systemic immune populations could complement radiological evaluation in lung cancer patients and help making informed 
decisions $[11,12,16]$. Quantification of systemic immunological parameters could be used to stratify patients before the start of immunotherapy [16], not only of immune populations but also cytokines such as IL-8 [17]. Moreover, information from immunological variables will shed light on the faulty immunological mechanisms present in nonresponders. This can open the door to therapeutic approaches that can successfully complement immune checkpoint blockade and increase response rates for lung cancer.

Financial \& competing interests disclosure

The immunomodulation group is funded by Asociación Española Contra el Cáncer (AECC, PROYE16001ESCO); Instituto de Salud Carlos III, Spain (FIS project grant PI17/02119), a 'Precipita' Crowdfunding grant (FECYT). D Escors is funded by a Miguel Servet Fellowship (ISC III, CP12/03114, Spain); M Zuazo I is supported by a scholarship from Universidad Pública de Navarra; H Arasanz is supported by a scholarship from AECC.

The authors have no other relevant affiliations or financial involvement with any organization or entity with a financial interest in or financial conflict with the subject matter or materials discussed in the manuscript apart from those disclosed.

No writing assistance was utilized in the production of this manuscript.

\section{Open access}

This work is licensed under the Attribution-NonCommercial-NoDerivatives 4.0 Unported License. To view a copy of this license, visit http://creativecommons.org/licenses/by-nc-nd/4.0/

\section{References}

1. Herbst RS, Baas P, Kim DW et al. Pembrolizumab versus docetaxel for previously treated, PD-L1-positive, advanced non-small-cell lung cancer (KEYNOTE-010): a randomised controlled trial. Lancet 387(10027), 1540-1550 (2016).

2. Horn L, Mansfiled AS, Szczęsna A et al. IMpower133 Study Group. First-line atezolizumab plus chemotherapy in extensive-stage small-cell lung cancer. N. Engl. J. Med. doi: 10.1056/NEJMoa1809064 (2018) (Epub ahead of print).

3. Reck M, Rodriguez-Abreu D, Robinson AG et al. Pembrolizumab versus chemotherapy for PD-L1-positive non-small-cell lung cancer. N. Engl. J. Med. 375(19), 1823-1833 (2016).

4. Champiat S, Ferrara R, Massard C et al. Hyperprogressive disease: recognizing a novel pattern to improve patient management. Nat. Rev. Clin. Oncol. doi:10.1038/s41571-018-0111-2 (2018) (Epub ahead of print).

5. Horn L, Spigel DR, Vokes EE et al. Nivolumab versus docetaxel in previously treated patients with advanced non-small-cell lung cancer: two-year outcomes from two randomized, open-label, Phase III trials (CheckMate 017 and CheckMate 057). J. Clin. Oncol. 35(35), 3924-3933 (2017).

6. Fehrenbacher L, Spira A, Ballinger M et al. POPLAR Study Group. Atezolizumab versus docetaxel for patients with previously treated non-small-cell lung cancer (POPLAR): a multicentre, open-label, Phase II randomised controlled trial. Lancet 387(10030), 1837-1846 (2016).

7. Rittmeyer A, Barlesi F, Waterkamp D et al. Atezolizumab versus docetaxel in patients with previously treated non-small-cell lung cancer (OAK): a Phase III, open-label, multicentre randomised controlled trial. Lancet 389(10066), 255-265 (2017).

8. Legrand FA, Gandara DR, Mariathasan S et al. Association of high tissue TMB and atezolizumab efficacy across multiple tumor types. $J$. Clin. Oncol. 36(15), 12000-12000 (2018).

9. Pages F, Mlecnik B, Marliot F et al. International validation of the consensus immunoscore for the classification of colon cancer: a prognostic and accuracy study. Lancet 391(10135), 2128-2139 (2018).

10. Sacdalan DB, Lucero JA, Sacdalan DL. Prognostic utility of baseline neutrophil-to-lymphocyte ratio in patients receiving immune checkpoint inhibitors: a review and meta-analysis. Onco. Targets Ther. 11, 955-965 (2018).

11. Kamphorst AO, Pillai RN, Yang S et al. Proliferation of PD-1+ CD8 T cells in peripheral blood after PD-1-targeted therapy in lung cancer patients. Proc. Natl Acad. Sci. USA 114(19), 4993-4998 (2017).

12. Krieg C, Nowicka M, Guglietta $S$ et al. High-dimensional single-cell analysis predicts response to anti-PD-1 immunotherapy. Nat. Med. 24(2), 144-153 (2018).

13. Gato-Canas M, Zuazo M, Arasanz H et al. PDL1 signals through conserved sequence motifs to overcome interferon-mediated cytotoxicity. Cell Rep. 20(8), 1818-1829 (2017).

14. Karwacz K, Bricogne C, Macdonald D et al. PD-L1 co-stimulation contributes to ligand-induced T cell receptor down-modulation on CD8(+) T cells. EMBO Mol. Med. 3(10), 581-592 (2011).

15. Kreiter S, Vormehr M, Van De Roemer N et al. Mutant MHC class II epitopes drive therapeutic immune responses to cancer. Nature 520(7549), 692-696 (2015). 
16. Zuazo M, Arasanz H, Fernandez-Hinojal G et al. Highly differentiated CD4 T cells unequivocally identify primary resistance and risk of hyperprogression to PD-L1/PD-1 immune checkpoint blockade in lung cancer. bioRxiv doi:10.1101/320176 (2018) (Epub ahead of print).

17. Sanmamed MF, Perez-Gracia JL, Schalper KA et al. Changes in serum interleukin-8 (IL-8) levels reflect and predict response to anti-PD-1 treatment in melanoma and non-small-cell lung cancer patients. Ann. Oncol. 28(8), 1988-1995 (2017). 